\title{
CDHI (E-Cadherin) Mutation and Gastric Cancer: Genetics, Molecular Mechanisms and Guidelines for Management
}

This article was published in the following Dove Press journal: Cancer Management and Research

\section{Santosh Shenoy (D)}

Clinical Associate Professor of Surgery, Department of Surgery, Kansas City VA Medical Center, University of Missouri Kansas City, Kansas City, MO 64I28, USA and Cancer Biology and Therapeutics, HMS High-Impact Cancer Research (HI-CR) Program, Harvard Medical School 2018-2019, Boston, MA 02II5, USA
Correspondence: Santosh Shenoy Department of Surgery, Kansas City VA Medical Center, University of Missouri Kansas City, 480I E Linwood Blvd, Kansas City, MO 64128, USA

Tel + |816 86| 4700 Ext 5543|

Email shenoy2009@hotmail.com
Introduction: Germline mutation in CDH1 (E-cadherin) tumor suppressor gene is associated with hereditary diffuse gastric cancer (HDGC) and lobular breast cancers (LBC). E-Cadherin protein is necessary for physiological signaling pathways, such as cell proliferation, maintenance of cell adhesion, cell polarity and epithelial-mesenchymal transition. Dysregulation leads to tumor proliferation, invasion, migration and metastases. We review current perspectives in CDH1 genetics with molecular mechanisms and also discuss management strategies for this aggressive form of gastric cancer.

Methods: Relevant articles from PubMed/Medline and Embase (1994-2019) were searched and collected using the phrases "Hereditary diffuse gastric cancer, Familial gastric cancer, CDH1 mutation, E-Cadherin, Lobular breast cancer, Prophylactic total gastrectomy".

Results: Current guidelines suggest maintaining a high degree of suspicion of hereditary etiology and recommend testing for $C D H 1$ mutations in patients with familial clustering of HDGC and LBC, especially onset at an early age (before 40 years). In families lacking CDH1 mutations but with high suspicion for hereditary predisposition, testing of CTNNA1 and other closely related HDGC susceptibility genes could be considered. Prophylactic total gastrectomy is recommended for individuals with identified pathogenic germline variants. Endoscopic surveillance with biopsies is recommended for those choosing to delay prophylactic gastrectomy.

Conclusion: Mutation or transcriptional silencing of the $\mathrm{CDH} 1$ gene is associated with familial diffuse gastric cancer. Further studies on the expression and the alteration in the proteins in the E-cadherin pathways may serve as biomarkers for early detection; stratify risk and selection of appropriate therapy in these families. Until then prophylactic total gastrectomy is recommended for individuals with $\mathrm{CDH} 1$ mutations and family history of diffuse gastric cancer. Endoscopic surveillance and biopsies by experienced gastroenterologists is recommended for those choosing not to have prophylactic gastrectomy and in individuals with $\mathrm{CDH1}$ variants.

Keywords: diffuse gastric cancer, $C D H 1$ gene, E-cadherin functions, lobular breast carcinoma

\section{Introduction}

Gastric cancer (GC) is an aggressive malignancy and a common cause of cancer-related deaths worldwide. An estimated 27,510 new cases will be diagnosed in the USA in 2019 with a mortality of 11,140 cases. ${ }^{1}$ The overall 5 -year survival rate for all types and stages of gastric cancer in the United States was 31\% (2008-2016) with a range of 68\% in patients with localized disease to $5 \%$ in patients with distant metastases. ${ }^{1}$

Gastric cancers are classified into two main histological types. ${ }^{2,3}$ The majority of gastric cancers are sporadic in nature, intestinal-type adenocarcinoma. Risk factors 
include infection with Helicobacter pylori, smoking, excessive alcohol consumption and a diet rich in salted and smoked foods. The incidence of sporadic distal gastric cancers associated with $H$. pylori is declining in the USA; however, its worldwide incidence seems to be on the rise.

Diffuse gastric cancers (DGC) also referred to as signet ring carcinoma, occur less frequently and has a worse prognosis due to infiltrative growth pattern (linitis plastica). Patients may present without a distinct endoluminal mass and tend to metastasize early.

A subset of patients with diffuse gastric cancers are associated with hereditary gastric cancer syndromes (HDGC) due to heterozygous germline mutations in the E-cadherin (E-cad also known as $\mathrm{CDHl}$ ) gene. The incidence of HDGC due to germline $\mathrm{CDH1}$ mutation ranges from $1 \%$ to $3 \%{ }^{4-6}$

HDGC has an autosomal dominant mode of inheritance. In $30-50 \%$ of the patients with HDGCs, the cause is mutated germline alleles of E-cadherin (CDH1). In the remaining families, the factors driving susceptibility remain unknown. ${ }^{4-6}$

This familial clustering of diffuse gastric cancer was initially observed and described in 1964, in a multigenerational Maori family from New Zealand. Becker et al in 1994 were the first to report the evidence of association of E-cad mutation with diffuse gastric cancer. ${ }^{7}$ In 1998, the E-cad germline gene mutation was identified and confirmed as the cause and association with hereditary gastric cancer in these Maori families. Subsequently, similar $C D H 1$ germline mutations were reported in diffuse gastric cancer in families of North European origin. ${ }^{4,5}$

\section{E-Cadherin Structure and Molecular}

\section{Function}

The $C D H 1$ gene is a tumor suppressor gene and is located on chromosome 16q22.1. This gene transcribes a $120-\mathrm{kDa}$ protein called E-cadherin. E-cadherin belongs to a family of highly conserved transmembrane glycoproteins called cadherin's whose function is to assist with calcium-dependent cell adhesion to form organized tissues by complexing with another set of cytosolic proteins called catenins.

E-cadherin glycoprotein consists of three structural domains: a single transmembrane domain, bridging the cytosolic domain, to the extracellular calcium-dependent domain consisting of five tandem repeats (Figure 1). The extracellular motif binds to a homophilic cadherin molecule from adjacent cells and this adhesion requires calcium ions which acts at a hinge and prevents the domain from flexing and provides it rigidity. The cytoplasmic tail of the E-cadherin protein interacts with filaments of cytoskeleton: actin through a set of adaptor proteins called catenin's (p120, $\beta$-catenin, and $\alpha$ catenin). This structure provides the cell stability and architecture and also inhibits individual cell motility. ${ }^{8}$

\section{E-Cadherin and Signaling Pathways in Cancer}

The exact mechanism of $\mathrm{CDH} 1$ mutations in carcinogenesis continues to be investigated. The role of E-cadherin in specific developmental processes as well as its function during carcinogenesis is reflected in the complex interplay

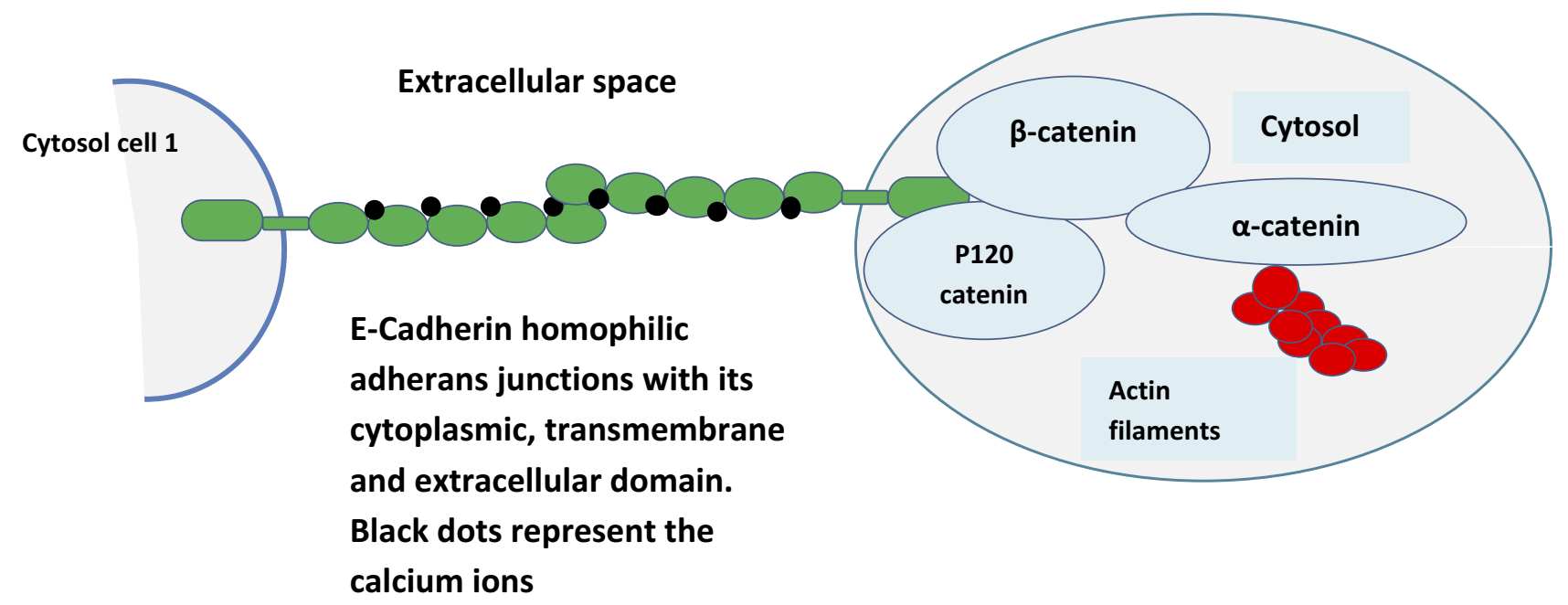

Figure I Structure and function of E-Cadherin-catenin complex (Adherens Junction). E-cadherin glycoprotein consists of three structural domains, a single transmembrane domain bridging the cytosolic domain to the extracellular calcium-dependent domain consisting of five tandem repeats (all domains are represented in green color and block dots represent calcium ions). The extracellular motif binds to homophilic cadherin molecule from adjacent cells and this adhesion requires calcium ions which acts at a hinge and prevents the domain from flexing and provides it rigidity. The cytoplasmic tail of the E-cadherin protein interacts with filaments of cytoskeleton: actin through a set of adaptor proteins called catenin's ( $\mathrm{P}$ |20, $\boldsymbol{\beta}$-catenin, and $\boldsymbol{\alpha}$-catenin). This structure provides the cell stability and architecture and also inhibits individual cell motility. 
of various signaling pathways. The E-cadherin-catenin complex functions in cell-cell adhesion mechanisms and also transduces signals to the nucleus and to the cytoskeleton, either directly or through a complex interplay with other pathways such as $\beta$-catenin, RhoGTPase, NF-kB and EGFR signaling in an adhesion-independent manner.

CDH1 gene mutation and its associated protein E-Cadherin (E-cad) loss lead to the process of EMT (epithelial-mesenchymal transition) whereby the cell loses its cellcell adhesion capabilities and apical polarity. In addition, important signaling interactions between E-cad and other cellular pathways include RTK/EGFR/MAPK pathway, $\mathrm{P}-120 /$ Rho/RAC pathway, and $\beta$-catenin/Wnt pathway and are described below. (Figure 2) (-28 $^{9}$ Genetic or epigenetic alterations in E-cadherin lead to alterations in epithelial cell-cell adhesion and cell structure, aberrant stromal interactions, as well as altered cell migration and signaling, thus promoting tumorigenesis. $^{9-28}$

\section{E-cad/ $\beta$-catenin/Wnt pathway}

$\beta$ catenin plays a central role as an adaptor protein linking E-cadherin to the actin cytoskeleton in cell-cell adhesion. It also remains a key component in the Wnt signaling. Under physiological conditions, cytoplasmic $\beta$-catenin remains in an inactive state by being bound to the APC/ GSK3 $\beta /$ Axin/CK1 degradation complex and undergoes phosphorylation for ubiquitination. Wnt signaling inhibits this degradative process by phosphorylating and inhibiting the GSK $3 \beta$ complex. This raises the critical threshold of $\beta$ catenin in the cytoplasm required to translocate into the nucleus. Under permissive conditions which amplify aberrant Wnt signaling such as paracrine factors from tumor environment, cytokines from stromal cells and TNF- $\alpha$ from macrophages, $\beta$-catenin translocates into the nucleus and binds to TCF-4/LEF-1 proteins to induce Wnt target genes such as c-Myc, cyclins, MMP. This leads to uncontrolled cell proliferation and growth. ${ }^{9-14}$

In the absence of E-cadherin, un-sequestered $\beta$-catenin released from the membrane-bound cadherin-catenin complex leads to excess cytoplasmic $\beta$-catenin. It has been demonstrated that $\beta$-catenin uses the same binding interface to engage TCF and E-cadherin ligands and cadherins have a superior binding affinity. There is a suggestion that as E-cad protein is lost, there is excess un-sequestered cytoplasmic $\beta$-catenin that escapes degradation and enters the nucleus to bind to TCF and activate Wnt pathway. In addition to activating downstream Wnt associated genes, it is also demonstrated that nuclear translocation of $\beta$-catenin represses PTEN expression. PTEN is a tumor suppressor and a critical regulator of AKT/MTOR pathway. Thus, the carefully balanced $\mathrm{Wnt} / \beta$-catenin/E-cad functioning is tipped in favor of uncontrolled cell proliferation promoting oncogenesis $^{9-15}$ (Figure 2).

\section{E-Cad/EGFR/RAS/RAF/MEK pathway}

Another function of E-cadherin is through its colocalization with EGFR at cell-cell contact and inhibiting EGFR pathways. Mutation of E-cadherin is associated with ligand-dependent activation of EGFR and downstream effectors through RAS/RAF/MEK pathways and also other pro-tumorigenic pathways such as FAK/c-Src and $\mathrm{PI} 3 \mathrm{~K} / \mathrm{AKT} / \mathrm{MTOR}$ pathway thus contributing to enhanced cell proliferation and motility. In addition, E-cad loss is associated with increased nuclear translocation of $\beta$-catenin into the nucleus as described earlier. This translocation of $\beta$-catenin represses PTEN expression. PTEN is a tumor suppressor and a critical regulator of AKT/MTOR pathway. ${ }^{9,10,15-20}$ (Figure 2).

\section{E-cad/P-I 20/Rho/MAPK pathway}

The Rho family of GTPases belongs to the RAS superfamily. They act as binary molecular switches and regulate many aspects of intracellular cytoskeleton dynamics, motility and cell polarity, cell proliferation. Normally, when E-cad protein is lost the cells undergo apoptosis through a process called anoikis, a form of programmed cell death occurring after cellular detachment and loss of cell architecture and polarity. Functional analysis of activated RhoA mutations suggests that activated RhoA inhibits anoikis. Other mechanism of RhoA activation occurs indirectly through adhesion-dependent interaction with the $\mathrm{p} 120$ protein. Besides having a physiological role in adherens junction as described above, p120 protein (Figure1) binds E-cadherin and stabilizes adherens junctions and suppresses both RhoA and NF-kB pathways. However, upon E-cadherin silencing, this negative regulation of RhoA is lost and p120 promotes cell growth by activating and modulating RhoGTPase and thus activating RAS/RAC/ MAPK activity and also through Rho mediated activation of inflammatory NF-kB pathways (BCL, IL-6, TNF). Although $H$. pylori is associated with intestinal type of gastric carcinoma; it may be a confounding factor in diffuse gastric cancer. It is demonstrated that $\mathrm{Cag}$ A (cytotoxic-associated gene A), secreted by $H$. pylori 


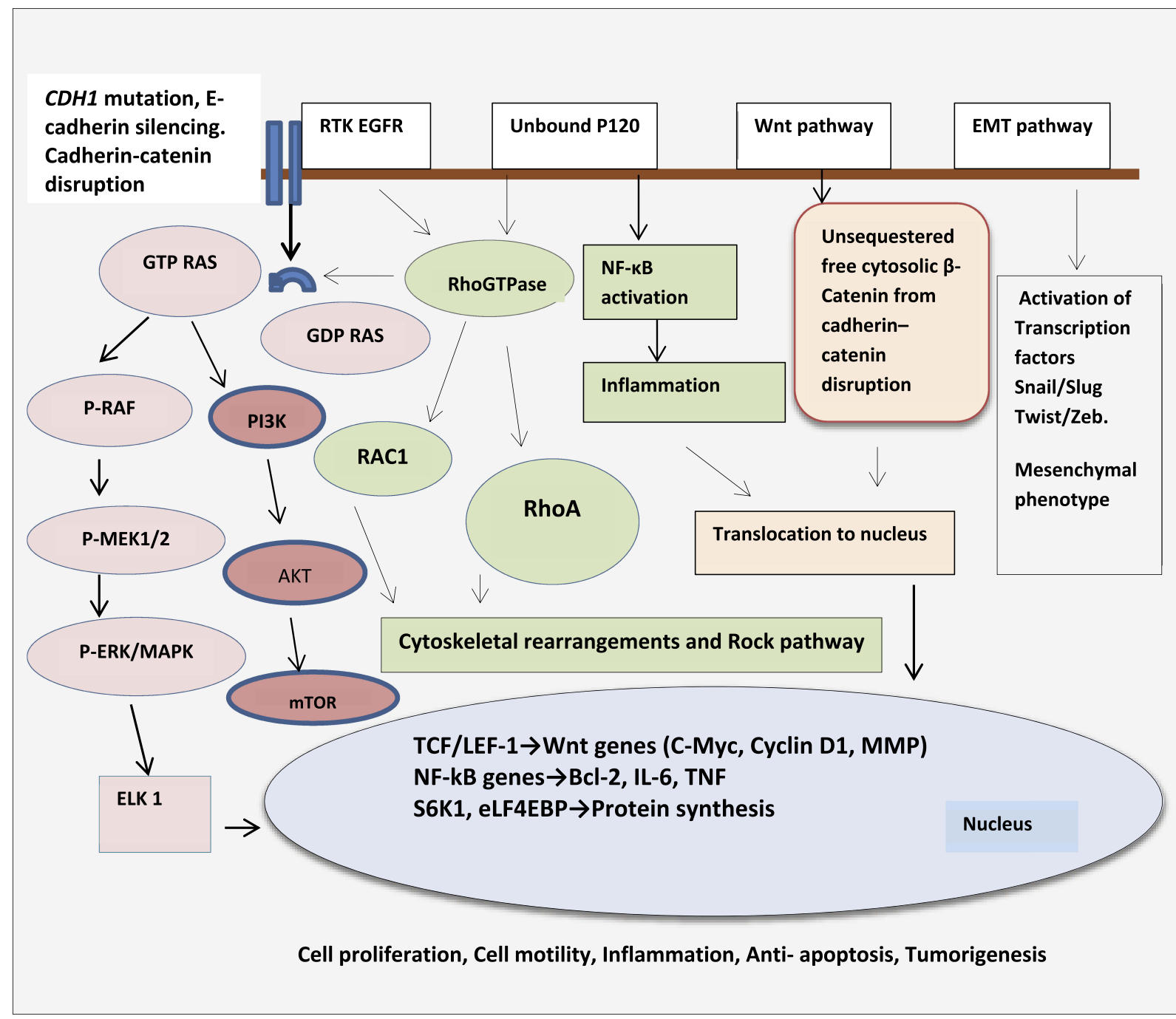

Figure 2 Signaling pathways and cross talk, regulated by E-cadherin. E-cad/ $\boldsymbol{\beta}$-catenin/Wnt pathway : $\boldsymbol{\beta}$ catenin plays a central role as an adaptor protein linking E-cadherin to the actin cytoskeleton in cell-cell adhesion. It also remains a key component in the Wnt signaling. Under physiological conditions, cytoplasmic $\boldsymbol{\beta}$-catenin remains in an inactive state by being bound to the APC/GSK3/Axin/CKI degradation complex and undergoes phosphorylation for ubiquitination. Wnt signaling inhibits this degradative process by phosphorylating and inhibiting the GSK3 $\beta$ complex. This raises the critical threshold of $\beta$-catenin in the cytoplasm required to translocate into the nucleus. Under permissive conditions which amplify aberrant $W$ nt signaling such as paracrine factors from tumor environment, cytokines from stromal cells and TNF- $\alpha$ from macrophages, $\boldsymbol{\beta}$-catenin translocates into the nucleus and binds to TCF-4/LEF-I proteins to induce Wnt target genes such as c-Myc, cyclins, MMP. This leads to uncontrolled cell proliferation and growth. ${ }^{9-14}$ In the absence of E-cadherin, un-sequestered $\beta$-catenin released from the membrane-bound cadherin-catenin complex leads to excess cytoplasmic $\boldsymbol{\beta}$-catenin. It has been demonstrated that $\boldsymbol{\beta}$-catenin uses the same binding interface to engage TCF and E-cadherin ligands and cadherins have a superior binding affinity. There is a suggestion that as $\mathrm{E}$-cad protein is lost there is excess un-sequestered cytoplasmic $\boldsymbol{\beta}$-catenin that escapes degradation and enters the nucleus to bind to TCF and activate Wnt pathway. In addition to activating downstream Wnt associated genes, it is also demonstrated nuclear translocation of $\beta$-catenin represses PTEN expression. PTEN is a tumor suppressor and a critical regulator of AKT/MTOR pathway. Thus, the carefully balanced Wnt/ $\beta$-catenin/E-cad functioning is tipped in favor of uncontrolled cell proliferation promoting oncogenesis ${ }^{9-15}$ (Figure 2). E-cad/EGFR/RAS/RAF/MEK: Another function of E-cadherin is through its co-localization with EGFR at cell-cell contact and inhibiting EGFR pathways. Mutation of E-cadherin is associated with ligand-dependent activation of EGFR and downstream effectors through RAS/RAF/MEK pathways and also other pro-tumorigenic pathways such as FAK/c-Src and PI3K/AKT/MTOR pathway thus contributing to enhanced cell proliferation and motility. In addition, E-cad loss is associated with increased nuclear translocation of $\boldsymbol{\beta}$-catenin into the nucleus as described earlier. This translocation of $\boldsymbol{\beta}$-catenin represses PTEN expression. PTEN is a tumor suppressor and a critical regulator of AKT/MTOR pathway, 10, $15-20$ (Figure 2). E-cad/P-I20/Rho/MAPK pathway: The Rho family of GTPases belongs to the RAS superfamily. They act as binary molecular switches and regulate many aspects of intracellular cytoskeleton dynamics, motility and cell polarity, cell proliferation. Normally, when E-cad protein is lost the cells undergo apoptosis through a process called anoikis, a form of programmed cell death occurring after cellular detachment and loss of cell architecture and polarity. Functional analysis of activated RhoA mutations suggests that activated RhoA inhibits anoikis. Other mechanism of RhoA activation occurs indirectly through adhesion-dependent interaction with the pl 20 protein. Besides having a physiological role in adherens junction as described above, p 120 protein (Figure I) binds E-cadherin and stabilizes adherens junctions and suppresses both RhoA and NF-kB pathways. However, upon E-cadherin silencing, this negative regulation of RhoA is lost and p 120 promotes cell growth by activating and modulating RhoGTPase and thus activating RAS/RAC/MAPK activity and also through Rho mediated activation of inflammatory NF-kB pathways (BCL, IL-6, TNF). Although $\mathrm{H}$. pylori is associated with intestinal type of gastric carcinoma; it may be a confounding factor in diffuse gastric cancer. It is demonstrated that $\mathrm{Cag}$ A (cytotoxic-associated gene $\mathrm{A}$ ), secreted by $\mathrm{H}$. pylori may cause epigenetic silencing of the E-cad gene, thus activate Rho through GRB/SHP-2 and downstream RAF/MEK/ERK pathway ${ }^{11}, 12,21-23,26-28$ (Figure 2). E-cad/snail, slug, twist and Zeb-I pathway :E-cadherin also play an important developmental role in EMT transition by suppression of transcription factors: snail, slug, twist and Zeb-I. These factors are implicated in the differentiation of epithelial cell into mesenchymal cells (EMT). Loss of E-cadherin protein promotes epithelial to mesenchymal cell (EMT) transition and thus loss of cell polarity and activation of motility. Snail, Twist and Zeb-I, previously known to cause E-cadherin repression, were among the transcription factors up-regulated following E-cadherin loss. This loss of cellular architecture leads to a migratory, mesenchymal phenotype important in metastatic disease. Cells lose cytokeratin and E-cad markers and gain other mesenchymal markers such as vimentin and N-cadherin. Loss of E-cad protein could be due to germline mutation as observed in HDGC or due to overexpression of transcription repressor (snail/slug/twist/zeb) factors by advanced carcinomas which downregulates E-cad expression. This phenomenon is also called transcriptional silencing. This leads to invasion and metastases ${ }^{10,14,18,24}$ (Figure 2). 
may cause epigenetic silencing of the E-cad gene, thus activates Rho through GRB/SHP-2 and downstream RAF/ MEK/ERK pathway ${ }^{11,12,21-23,26-28}$ (Figure 2).

\section{E-Cad/Snail, Slug, Twist and Zeb-I pathway}

E-cadherin also plays an important developmental role in EMT transition by suppression of transcription factors: snail, slug, twist and Zeb-1. These factors are implicated in the differentiation of epithelial cell into mesenchymal cells (EMT). Loss of E-cadherin protein promotes epithelial to mesenchymal cell (EMT) transition and thus loss of cell polarity and activation of motility. Snail, Twist and Zeb-1, previously known to cause E-cadherin repression, were among the transcription factors up-regulated following E-cadherin loss. This loss of cellular architecture leads to a migratory, mesenchymal phenotype important in metastatic disease. Cells lose cytokeratin and E-cad markers and gain other mesenchymal markers such as vimentin and N-cadherin. Loss of E-cad protein could be due to germline mutation as observed in HDGC or due to overexpression of transcription repressor (snail/slug/twist/zeb) factors by advanced carcinomas which downregulates E-cad expression. This phenomenon is also called transcriptional silencing. This leads to invasion and $\operatorname{metastases}^{10,14,18,24}$ (Figure 2).

\section{E-Cadherin Genetics and Testing}

Truncating mutation in $C D H 1$ gene leads to production of abnormally short, nonfunctional protein. This leads to disruption of cadherin-catenin's complex and loss of cell adhesion and results in increased cell motility, uncontrollable cell growth, division and increasing the metastatic ability of the tumor as described above. 9,10,26,29-31

The genetic alterations are scattered over the entire gene length in HDGC. Eighty percent of these CDHI germline mutations are truncating, resulting in complete loss of E-cad protein due to occurrence of premature stop codons. Twenty percent of the mutations are missense type resulting in an E-cad protein with an amino acid substitution. The functional impact of missense type of mutation is not clear and remains under investigation. ${ }^{25,32-35}$

$\mathrm{CDH1}$ is a tumor suppressor gene and therefore both alleles have to be silenced for loss of protein. A second hit is required for inactivation of the gene and tumorigenesis. The mechanisms by which the second allele of $C D H 1$ is inactivated may include hyper-methylation of the $\mathrm{CDH} 1$ promoter site causing epigenetic silencing, somatic mutation and loss of heterozygosity. ${ }^{25,32-35}$

The penetrance of HDGC gene is incomplete. Approximately $30 \%-50 \%$ of HDGC families may harbor this mutation. ${ }^{36-38}$ In a recent series of mutation carriers, the cumulative incidence of gastric cancer was 70\% $(95 \%$ CI, 59\%-80\%) for males and 56\% (95\% CI, 44\%-69\%) for females and the risk of lobular breast cancer was $42 \%(95 \%$ CI, 23\%-68\%). In the same study patients who tested negative for $C D H 1$ mutation had mutations noted in closely related HDGC susceptibility genes such as CTNNA1, BRCA2, STK11, SDHB, PRSS1, ATM, MSR1, and PALB2. ${ }^{39}$

The majority of these patients present with diffuse gastric cancer in their mid-thirties (range 14-69 years) and are more commonly reported in females. In contrast, sporadic, intestinal type of gastric cancer presents in men and in their fifth to seventh decade. ${ }^{1,37,40}$ The risk of lobular breast cancer (LBC) in these families approaches $42 \%$ by age 80 years, compared with the $12.5 \%$ lifetime risk for sporadic breast cancer. ${ }^{1,6,39-42}$

Based on the guidelines by International consortium of gastric cancer experts (IGCLC 2015) the diagnosis of $C D H 1$ mutation is suspected in the family of HDGC proband in the presence of two or more family members diagnosed with gastric cancer at any age with one confirmed DGC, family members diagnosed with DGC before the age of 40 years and members with diagnoses of both DGC and LBC with one diagnosis before the age of 50 years. In addition, $C D H 1$ mutation should be suspected and tested in families with bilateral or familial LBC occurring prior to the age of 50 . Other abnormalities associated with $C D H 1$ mutation are patients with DGC and cleft lip/palate, and those with precursor lesions for signet ring cell carcinoma. ${ }^{43}$

Genetic counselling is critical and an absolute prerequisite in the care of patients with $C D H 1$ mutation carriers. Because of the life-altering consequences of gastric surgery and breast diseases, family members should be offered genetic testing when they reach adulthood. Genetic screening may have long-term medical, psychological and insurance-related consequences and therefore counseling should be provided before testing, so that affected individuals can make informed decisions to get tested. Family members who test positive for mutations may then choose either, surveillance gastric-endoscopy or prophylactic gastrectomy to prevent gastric cancer. ${ }^{4,5,43}$

Sequence analysis of $C D H 1$ gene may be performed on blood or tissue sample first, followed by gene-targeted deletion or duplication analysis. Multigene analysis may be 
considered for other closely related HDGC susceptibility genes such as CTNNA1, MAPK, BRCA2, STK11, SDHB, PRSS1, ATM, MSR1, and PALB2, if CDH1 mutation analysis is tested negative. ${ }^{39,43,44}$ These newly identified gene mutations are rare and of inadequate sample size to draw any specific conclusions. $\mathrm{CDH1}$ analysis should include entire open reading frame, intron-exon boundaries, and copy number analysis to detect intragenic exon deletions or duplications. Approximately 155 different germline mutations have been described and the majority of these pathogenic mutations are truncating. About $20 \%$ of these CDH1 mutations may be missense mutations also known as VUS (variants of unknown significance) and the consequence of which is currently not clear. These specimens and family data should be referred for bioinformatics and image analysis, protein quantification, in-silico studies, expression analysis, and a series of tests performed in transgenic cells in-vitro culture. Useful information may be obtained regarding possible pathogenic effects of these CDH1 VUS and in determining the need for total prophylactic gastrectomy. ${ }^{35,39,43,44}$

In addition to the mutations described, E-cad silencing may also occur through other mechanisms including overexpression of transcription repressors, alterations of microRNAs (miRNAs), deregulation of protein trafficking, and aberrant post-translational glycosylation regulation of the protein. ${ }^{10,25,35,45,46}$

\section{Cancer Screening and Surveillance}

The average age for clinical presentation in HDGC is 38 years and is generally asymptomatic in majority of the patients. When specific symptoms do appear, the disease is typically in advanced metastatic stages and has poor prognosis. Analysis on the prophylactic post-gastrectomy specimens in $\mathrm{CDH} 1$ mutant variants describes multifocal, isolated nests of neoplastic signet ring cells at the base of the glands along with pagetoid infiltrative pattern of spread under a histologically normal-appearing mucosa. In advanced cases, the stomach may appear thickened and rigid, a phenomenon known as linitis plastica. ${ }^{47}$

Patients with positive pathogenic germline mutation should consider prophylactic total gastrectomy regardless of endoscopic findings. For patients who decline prophylactic gastrectomy, screening and surveillance upper endoscopy following Cambridge protocol should be offered. Screening should begin $5-10$ years prior to the youngest family member's diagnosis. The current recommendations are semiannual or annual, detailed 30 min-high-definition white light upper endoscopy with biopsy of any visible suspicious lesions including pale mucosa. In addition, multiple random deep biopsies obtained from pre-pyloric area, antrum, transitional zone, body, fundus and cardia. A minimum of 30 biopsies is recommended. ${ }^{43,48-50}$ The stomach should be inflated to check for ease of distention. If stomach appears rigid and is suspicious of linitis plastica an endoscopic ultrasonography or a CT scan should be performed to evaluate the layers of the wall. ${ }^{4-50}$ Images are recorded for future comparisons. In spite of advanced endoscopy techniques, the detection of cancerous foci in $\mathrm{CDHl}$ carriers is poor. Post-gastrectomy evaluation of surgical specimens demonstrated signet ring cancer in $45-60 \%$ of those with negative endoscopic evaluations. ${ }^{50}$ Other tools used in the past such as chromo-endoscopy with Congo red and methylene blue dye are not recommended due to concerns of toxicity with these dyes. PET CT scans although not very useful for early lesions, may provide evidence of lymph nodes and advanced gastric wall infiltration (linitis plastica). ${ }^{43,51}$

Given the rarity of lobular breast cancer (LBC) in general population, there is insufficient data regarding best surveillance practices for early detection of breast cancer in female patients with CDH1 mutation carriers. LBC often presents as sheets of malignant cells and do not form a well-defined mass as compared with invasive ductal cancer. The sensitivity of a mammogram for detecting LBC is therefore suboptimal. ${ }^{52,53}$

Female's carriers with $\mathrm{CDH} 1$ gene mutation should closely follow with a breast oncologist and breast surgeon for yearly clinical breast exams and screening for lobular breast cancer. Screening recommendations include bilateral breast MRIs beginning at age 30. Chemoprevention of high-risk patients per the established national guidelines using selective estrogen receptor modulators or aromatase inhibitors could be considered. Some patients may consider preventative mastectomy as an option, but this is not routinely recommended for $C D H 1$ mutation carriers. ${ }^{19,29,42,43,52,53}$

\section{Prophylactic Total Gastrectomy}

Given the asymptomatic nature of DGC and erratic growth pattern, it is possible the tumor may remain dormant and then progress rapidly. It is estimated that the risk for DGC to be $1 \%$ by the age of 20 years and increases to $4 \%$ by the age of 30 years in these carriers. ${ }^{40}$

Once symptoms appear the prognosis is poor, and most patients are diagnosed with advanced disease. Further, these mutation carriers have high but variable, unpredictable penetrance. Prophylactic total gastrectomy is an appropriate option for mutation carriers. 
Lewis et al were among the first surgeons to recommend prophylactic total gastrectomy for asymptomatic patients with $C D H 1$ mutations. Their recommendation was based upon the finding of occult diffuse cancer in post-gastrectomy specimens of six asymptomatic members of $\mathrm{CDH} 1$ mutated families. ${ }^{36}$

Subsequently, other authors published similar data. Huntsman et al described in their series of 5 patients from families with $\mathrm{CDH} 1$ mutation who underwent prophylactic total gastrectomy. All patients showed evidence of HDGC on final pathology that failed to show on preoperative endoscopy. ${ }^{47}$ Similarly, Norton et al reported occult diffuse gastric cancer in six patients with $\mathrm{CDH1}$ mutation who underwent prophylactic total gastrectomy. All of their resected gastrectomy specimens had presence of multifocal $\mathrm{T} 1$ invasive DGC without lymph node involvement. ${ }^{54}$

In a recent series of 174 gastrectomy specimens from asymptomatic carriers with $\mathrm{CDHI}$ mutation, preoperative endoscopic biopsies were positive for cancer only in $28.3 \%$ of specimens. A macroscopic lesion was noted in $11.7 \%$ of the specimens. Subsequently, intraepithelial lesions and/or intra-mucosal signet ring cell carcinoma were present in $87.9 \%$ of the stomach specimens. Helicobacter pylori infection was found in $23.4 \%$ of the cases. ${ }^{55}$

The optimal age to perform prophylactic gastrectomy has to be individualized however most authors recommend it be performed during early adulthood between 20 and 30 years of age. ${ }^{39,40,43,47,56}$ Female carriers wanting pregnancy may have to delay their operations to prevent complications from nutritional and metabolic deficiencies.

\section{Pre-Procedural and Operative Considerations}

Total gastrectomy carries a high morbidity with considerable impact on psychological, physiological and metabolic wellbeing. A multidisciplinary team approach is necessary. $\mathrm{CDH1}$ mutation carriers should meet with clinical geneticist, psychologist, nutritionist, in addition to surgeon, and oncologists and should be supported in the post-operative period. Nutritional parameters such as serum albumin and prealbumin should be optimized. Blood sugar should be adequately controlled in diabetics. Metastatic disease has to be ruled out with appropriate imaging such a CT scan of the abdomen and pelvis. Screening colonoscopy is also recommended due to association of $C D H 1$ mutations with colon cancer. ${ }^{19,43,47}$

Total gastrectomy with removal of all gastric mucosa with Roux-en- Y esophagus-jejunal reconstruction, with or without a pouch is the most widely performed technique. ${ }^{36,54,57-59}$ The procedure is performed with an open or a laparoscopic/robotic techniques. Advantage of laparoscopic/robotic approach is reduced pain and faster recovery. ${ }^{58,59}$ Post-operative incidence of anastomotic leak, stenosis, morbidity, and length of stay is not statistically different when a stapled versus a hand-sewn anastomosis is performed. ${ }^{57-60}$

Frozen sections should be checked at the edges to ensure esophageal mucosa proximally and duodenal mucosa distally confirming total resection. Younger patients recover well as most are healthy without baseline medical diseases. The overall surgical mortality is less than $1 \%$ in experienced centers. ${ }^{31,36,54-60}$

The extent of lymph node dissection remains unclear. For prophylactic gastrectomy, before the onset of gastric cancer, the incidence of lymph node metastasis is less than 5\%. A D1 lymphadenectomy (peri-gastric lymph node stations, 1-6) may be adequate. For patients with confirmed gastric cancer, an extended D1 and D2 station (8-12) lymphadenectomy may be necessary. ${ }^{43,61,62}$

The complications from gastrectomy are more severe in older physiologically and nutritionally depleted patients. Untreated, HDGC may have $100 \%$ mortality due to eventual development of metastatic disease.

\section{Follow-Up and Quality of Life}

Total gastrectomy is associated with significant weight loss. Rapid weight loss occurs initially and is followed by weight stabilization in a year or two following surgery with an average $10-15 \%$ permanent weight loss. Most patients experience rapid intestinal transit, reflux, dumping syndrome, and diarrhea. ${ }^{39,43,63}$ These symptoms may be severe initially and improve and stabilize with time. Quality of life (QOL) studies indicate high association with anxiety, depression. ${ }^{63-65}$ Younger, male patients have better QOL and outlook compared with female and older patients. Extensive lymphadenectomy with D2 and D3 resections tend to have worse functional outcomes, but no significant effect on QOL. ${ }^{63-69}$

There is an increase in the risk of metabolic derangements due to loss of stomach acid and absorptive function. Common post-gastrectomy deficiencies include calcium, vitamin $\mathrm{D}$, iron and vitamin B12. Small bacterial overgrowth may occur which may further exacerbate nutritional deficiency and protein-calorie malnutrition. Post-gastrectomy patients require lifelong vitamin supplementations. ${ }^{36,43,67,68}$

Pregnancy after total gastrectomy is feasible and reported with normal outcomes. Pregnant carriers should 
be closely monitored for nutritional deficiencies in mother and, growth and development of the fetus. Genetic predisposition through preimplantation genetic diagnosis is an option and should be discussed with all female carriers or if their partners are $C D H 1$ carriers wishing to get pregnant. $^{43,70,71}$

\section{Conclusion}

E-cadherin and its associated signaling pathways play an important physiological role in maintaining cell-cell adhesions, architecture, motility and cell homeostasis. Dysregulation, mutation or transcriptional silencing of the $C D H 1$ gene leads to gastric carcinogenesis.

Further studies on the expression and the alteration in the proteins in the E-cadherin pathways may serve as promising biomarkers for early detection; stratify risk and selection of appropriate therapy in these families. Until then prophylactic total gastrectomy is recommended for individuals with $\mathrm{CDH} 1$ mutations and family history of diffuse gastric cancer. Endoscopic surveillance in experienced centers is recommended for those opting not to have prophylactic gastrectomy and in individuals with $C D H 1$ variants.

\section{Disclosure}

The author reports no conflict of interest in this work.

\section{References}

1. Surveillance, Epidemiology, and End Results (SEER) Program. SEER*stat database: statistics at a Glance: stomach cancer. Available From: http://www.seer.cancer.gov. Accessed July 13, 2019.

2. Lauren P. The two histological main types of gastric carcinoma: diffuse and so-called intestinal-type carcinoma. An attempt at a histo-clinical classification. Acta Pathol Microbiol Scand. 1965;64:31-49. doi:10.1111/apm.1965.64.1.31

3. Bosman FT, Carneiro F, Hruban RH, Theise ND. WHO Classification of Tumors of the Digestive System. Lyon: International Agency for research on Cancer; 2010:48-58.

4. Guilford P, Hopkins J, Harraway J, et al. E-cadherin germline mutations in familial gastric cancer. Nature. 1998;392(6674):402-405. doi: $10.1038 / 32918$

5. Gayther SA, Gorringe KL, Ramus SJ, et al. Identification of germ-line E-cadherin mutations in gastric cancer families of European origin. Cancer Res. 1998;58(18):4086-4089.

6. Corso G, Intra M, Trentin C, Veronesi P, Galimberti V. CDH1 germline mutations and hereditary lobular breast cancer. Fam Cancer. 2016;15(2):215-219. doi:10.1007/s10689-016-9869-5

7. Becker KF, Atkinson MJ, Reich U, et al. E-cadherin gene mutations provide clues to diffuse type gastric carcinomas. Cancer Res. 1994;54 (14):3845-3852.

8. Gall TM, Frampton AE. Gene of the month: E-cadherin (CDH1). J Clin Pathol. 2013;66(11):928-932. doi:10.1136/jclinpath-2013-201768

9. Gottardi CJ, Wong E, Gumbiner BM. E-cadherin suppresses cellular transformation by inhibiting beta-catenin signaling in an adhesion-independent manner. J Cell Biol. 2001;153(5):1049-1060. doi: $10.1083 /$ jcb.153.5.1049
10. Jeanes A, Gottardi CJ, Yap AS. Cadherins and cancer: how does cadherin dysfunction promote tumor progression? Oncogene. 2008;27(55):6920-6929. doi:10.1038/onc.2008.343

11. Kourtidis A, Ngok SP, Anastasiadis PZ. p120 catenin: an essential regulator of cadherin stability, adhesion-induced signaling, and cancer progression. Prog Mol Biol Transl Sci. 2013;116:409-432.

12. Bruner HC, Derksen PWB. Loss of E-Cadherin-dependent cell-cell adhesion and the development and progression of cancer. Cold Spring Harb Perspect Biol. 2018;10(3):a029330. doi:10.1101/cshperspect.a029330

13. Nelson WJ, Nusse R. Convergence of Wnt, beta-catenin, and cadherin pathways. Science. 2004;303(5663):1483-1487. doi:10.1126/ science. 1094291

14. Yang J, Weinberg RA. Epithelial-mesenchymal transition: at the crossroads of development and tumor metastasis. Dev Cell. 2008;14 (6):818-829. doi:10.1016/j.devcel.2008.05.009

15. Li D, Lo W, Rudloff U. Merging perspectives: genotype-directed molecular therapy for hereditary diffuse gastric cancer (HDGC) and E-cadherin-EGFR crosstalk. Clin Transl Med. 2018;7(1):7. doi:10.1186/s40169-018-0184-7

16. Qian X, Karpova T, Sheppard AM, McNally J, Lowy DR. E-cadherin-mediated adhesion inhibits ligand-dependent activation of diverse receptor tyrosine kinases. EMBO J. 2004;23 (8):1739-1748. doi:10.1038/sj.emboj.7600136

17. Bremm A, Walch A, Fuchs M, et al. Enhanced activation of epidermal growth factor receptor caused by tumor-derived E-cadherin mutations. Cancer Res. 2008;68(3):707-714. doi:10.1158/0008-5472.CAN-07-1588

18. Onder TT, Gupta PB, Mani SA, Yang J, Lander ES, Weinberg RA. Loss of E-cadherin promotes metastasis via multiple downstream transcriptional pathways. Cancer Res. 2008;68(10):3645-3654. doi:10.1158/0008-5472.CAN-07-2938

19. Figueiredo J, Melo S, Carneiro P, et al. Clinical spectrum and pleiotropic nature of CDH1 germline mutations. J Med Genet. 2019;56 (4):199-208. doi:10.1136/jmedgenet-2018-105807

20. Perrais M, Chen X, Perez-Moreno M, Gumbiner BM. E-cadherin homophilic ligation inhibits cell growth and epidermal growth factor receptor signaling independently of other cell interactions. Mol Biol Cell. 2007;18(6):2013-2025. doi:10.1091/mbc.e06-04-0348

21. Tan P, Yeoh KG. Genetics and molecular pathogenesis of gastric adenocarcinoma. Gastroenterology. 2015;149(5):1153-1162. doi:10.1053/j.gastro.2015.05.059

22. Nam S, Kim JH, Lee DH. RHOA in gastric cancer: functional roles and therapeutic potential. Front Genet. 2019;10:438. doi:10.3389/ fgene. 2019.00438

23. Cowell CF, Yan IK, Eiseler T, Leightner AC, Döppler H, Storz P. Loss of cell-cell contacts induces NF-kappaB via RhoA-mediated activation of protein kinase D1. J Cell Biochem. 2009;106 (4):714-728. doi:10.1002/jcb.22067

24. Cano A, Pérez-Moreno MA, Rodrigo I, et al. The transcription factor snail controls epithelial-mesenchymal transitions by repressing E-cadherin expression. Nat Cell Biol. 2000;2(2):76-83. doi:10.1038/35000025

25. Berx G, van Roy F. Involvement of members of the cadherin superfamily in cancer. Cold Spring Harb Perspect Biol. 2009;1(6): a003129. doi:10.1101/cshperspect.a003129

26. Carneiro P, Fernandes MS, Figueiredo J, Caldeira J, et al. E-cadherin dysfunction in gastric cancer-cellular consequences, clinical applications and open questions. FEBS Lett. 2012;586(18):2981-2989. doi:10.1016/j.febslet.2012.07.045

27. Adam AJ, Thorsson V, Shmulevich I; Cancer genome atlas research network, et al. Comprehensive molecular characterization of gastric adenocarcinoma. Nature. 2014;513(7517):202-209.

28. Ushiku T, Ishikawa $\mathrm{S}$, Kakiuchi $\mathrm{M}$, et al. RHOA mutation in diffuse-type gastric cancer: a comparative clinicopathology analysis of 87 cases. Gastric Cancer. 2016;19(2):403-411. doi:10.1007/ s10120-015-0493-0

29. Schrader KA, Masciari S, Boyd N, et al. Hereditary diffuse gastric cancer: association with lobular breast cancer. Fam Cancer. 2008;7(1):73-82. 
30. Suriano G, Oliveira C, Ferreira P, et al. Identification of $\mathrm{CDH} 1$ germline missense mutations associated with functional inactivation of the E-cadherin protein in young gastric cancer probands. Hum Mol Genet. 2003;12(5):575-582. doi:10.1093/hmg/ddg048

31. Lynch HT, Silva E, Wirtzfeld D, Hebbard P, Lynch J, Huntsman DG. Hereditary diffuse gastric cancer: prophylactic surgical oncology implications. Surg Clin N Am. 2008;88(4):759-778. doi:10.1016/j. suc.2008.04.006

32. Barber M, Murrell A, Ito Y, et al. Mechanisms and sequelae of E-cadherin silencing in hereditary diffuse gastric cancer. $J$ Pathol. 2008;216(3):295-306. doi:10.1002/path.2426

33. Oliveira C, Sousa S, Pinheiro H, et al. Quantification of epigenetic and genetic 2nd hits in $\mathrm{CDH} 1$ during hereditary diffuse gastric cancer syndrome progression. Gastroenterology. 2009;136(7):2137-2148. doi:10.1053/j.gastro.2009.02.065

34. Grady WM, Willis J, Guilford PJ, et al. Methylation of the CDH1 promoter as the second genetic hit in hereditary diffuse gastric cancer. Nat Genet. 2000;26(1):16-17. doi:10.1038/79120

35. Melo S, Figueiredo J, Fernandes MS, et al. Predicting the functional impact of $\mathrm{CDH} 1$ Missense mutations in hereditary diffuse gastric cancer. Int J Mol Sci. 2017;18:12. doi:10.3390/ijms18122687

36. Lewis FR, Mellinger JD, Hayashi A, et al. Prophylactic total gastrectomy for familial gastric cancer. Surgery. 2001;130(4):612-617.

37. Guilford P, Blair V, More H, Humar B. A short guide to hereditary diffuse gastric cancer. Hered Cancer Clin Pract. 2007;5(4):183-194. doi:10.1186/1897-4287-5-4-183

38. Lynch HT, Kaurah P, Wirtzfeld D, et al. Hereditary diffuse gastric cancer: diagnosis, genetic counseling, and prophylactic total gastrectomy. Cancer. 2008;112(12):2655-2663.

39. Hansford S, Kaurah P, Li-Chang H, et al. Hereditary diffuse gastric cancer syndrome: CDH1 mutations and beyond. JAMA Oncol. 2015;1 (1):23-32. doi:10.1001/jamaoncol.2014.168

40. Pharoah PD, Guilford P, Caldas C; International Gastric Cancer Linkage Consortium. Incidence of gastric cancer and breast cancer in CDH1 (E-cadherin) mutation carriers from hereditary diffuse gastric cancer families. Gastroenterology. 2001;121(6):1348-1353. doi:10.1053/gast.2001.29611

41. Brooks-Wilson AR, Kaurah P, Suriano G, et al. Germline E-cadherin mutations in hereditary diffuse gastric cancer: assessment of 42 new families and review of genetic screening criteria. $J$ Med Genet. 2004;41(7):508-517. doi:10.1136/jmg.2004.018275

42. Keller G, Vogelsang H, Becker I, et al. Diffuse type gastric and lobular breast carcinoma in a familial gastric cancer patient with an E-cadherin germline mutation. Am J Pathol. 1999;155(2):337-342. doi:10.1016/S0002-9440(10)65129-2

43. van der Post RS, Vogelaar IP, Carneiro F, et al. Hereditary diffuse gastric cancer: updated clinical guidelines with an emphasis on germline CDH1 mutation carriers. J Med Genet. 2015;52(6):361-374. doi:10.1136/jmedgenet-2015-103094

44. Fewings E, Larionov A, Redman J, et al. Germline pathogenic variants in PALB2 and other cancer-predisposing genes in families with hereditary diffuse gastric cancer without $\mathrm{CDH} 1$ mutation: a whole-exome sequencing study. Lancet Gastroenterol Hepatol. 2018;3(7):489-498. doi:10.1016/S2468-1253(18)30079-7

45. Carvalho S, Catarino TA, Dias AM, et al. Preventing E-cadherin aberrant $\mathrm{N}$-glycosylation at Asn-554 improves its critical function in gastric cancer. Oncogene. 2016;35(13):1619-1631. doi:10.1038/onc.2015.225

46. Ansari S, Gantuya B, Tuan VP, Yamaoka Y. Diffuse gastric cancer: a summary of analogous contributing factors for its molecular pathogenicity. Int J Mol Sci. 2018;19:8.

47. Huntsman DG, Carneiro F, Lewis FR, et al. Early gastric cancer in young, asymptomatic carriers of germline E-cadherin mutations. NEJM. 2001;344(25):1904-1909. doi:10.1056/NEJM200106213442504
48. Fitzgerald RC, Hardwick R, Huntsman D; International Gastric Cancer Linkage Consortium, et al. Hereditary diffuse gastric cancer: updated consensus guidelines for clinical management and directions for future research. J Med Genet. 2010;47(7):436-444. doi:10.1136/jmg.2009.074237

49. Syngal S, Brand RE, Church JM, Giardiello FM, Hampel HL, Burt RW; American College of Gastroenterology. ACG clinical guideline: genetic testing and management of hereditary gastrointestinal cancer syndromes. Am J Gastroenterol. 2015;110(2):223-262. doi:10.1038/ajg.2014.435

50. Kumar S, Long JM, Ginsberg GG, Katona BW. The role of endoscopy in the management of hereditary diffuse gastric cancer syndrome. World J Gastroenterol. 2019;25(23):2878-2886. doi:10.3748/wjg.v25.i23.2878

51. van Kouwen MC, Drenth JP, Oyen WJ, et al. [18F] Fluoro-2-deoxyD-glucose positron emission tomography detects gastric carcinoma in an early stage in an asymptomatic E-cadherin mutation carrier. Clin Cancer Res. 2004;10(19):6456-6459. doi:10.1158/1078-0432.CCR-04-0599

52. Dossus L, Benusiglio PR. Lobular breast cancer: incidence and genetic and non-genetic risk factors. Breast Cancer Res. 2015;17:37. doi:10.1186/s13058-015-0546-7

53. Knuttel FM, Menezes GL, van den Bosch MA, Gilhuijs KG, Peters NH. Current clinical indications for magnetic resonance imaging of the breast. J Surg Oncol. 2014;110(1):26-31. doi:10.1002/ jso. 23655

54. Norton JA, Ham CM, Van Dam J, et al. CDH1 truncating mutations in the E-cadherin gene: an indication for total gastrectomy to treat hereditary diffuse gastric cancer. Ann Surg. 2007;245(6):873-879. doi:10.1097/01.sla.0000254370.29893.e4

55. Rocha JP, Gullo I, Wen X, et al. Pathological features of total gastrectomy specimens from asymptomatic hereditary diffuse gastric cancer patients and implications for clinical management. Histopathology. 2018;73(6):878-886.

56. Blair V, Martin I, Shaw D, et al. Hereditary diffuse gastric cancer: diagnosis and management. Clin Gastroenterol Hepatol. 2006;4 (3):262-275. doi:10.1016/j.cgh.2005.12.003

57. Newman EA, Mulholland MW. Prophylactic gastrectomy for hereditary diffuse gastric cancer syndrome. J Am Coll Surg. 2006;202 (4):612-617. doi:10.1016/j.jamcollsurg.2005.12.017

58. Hackenson D, Edelman DA, McGuire T, Weaver DW, Webber JD. Prophylactic laparoscopic gastrectomy for hereditary diffuse gastric cancer: a case series in a single family. JSLS. 2010;14(3):348-352. doi:10.4293/108680810X12924466007449

59. Haverkamp L, van der Sluis PC, Ausems MG, et al. Prophylactic laparoscopic total gastrectomy with Jejunal pouch reconstruction in patients carrying a CDH1 germline mutation. J Gastrointest Surg. 2015;19(12):2120-2125. doi:10.1007/s11605-015-2963-4

60. Hyodo M, Hosoya Y, Hirashima Y, et al. Minimum leakage rate $(0.5 \%)$ of stapled esophagojejunostomy with sacrifice of a small part of the jejunum after total gastrectomy in 390 consecutive patients. Dig Sur. 2007;24(3):169-172. doi:10.1159/000102100

61. Schmidt B, Yoon SS. D1 versus D2 lymphadenectomy for gastric cancer. J Surg Oncol. 2013;107(3):259-264. doi:10.1002/jso.23127

62. Pantelis D, Hüneburg R, Adam R, et al. Prophylactic total gastrectomy in the management of hereditary tumor syndromes. Int J Colorectal Dis. 2016;31(12):1825-1833. doi:10.1007/s00384016-2656-9

63. Worster E, Liu X, Richardson S, et al. The impact of prophylactic total gastrectomy on health-related quality of life: a prospective cohort study. Ann Surg. 2014;260(1):87-93. doi:10.1097/ SLA.0000000000000446

64. Muir J, Aronson M, Esplen MJ, Pollett A, Swallow CJ. Prophylactic total gastrectomy: a prospective cohort study of long-term impact on quality of life. J Gastrointest Surg. 2016;20(12):1950-1958. doi:10.1007/s11605-016-3287-8 
65. Kaurah P, Talhouk A, MacMillan A, et al. Hereditary diffuse gastric cancer: cancer risk and the personal cost of preventive surgery. Fam Cancer. 2019. doi:10.1007/s10689-019-00133-9

66. Wu CW, Chio JM, Ko FS, et al. Quality of life after curative gastrectomy for gastric cancer in a randomized controlled trial. $\mathrm{Br}$ J Cancer. 2008;98:54-59. doi:10.1038/sj.bjc.6604097

67. Buhl K, Lehnert T, Schlag P, Herfarth C. Reconstruction after gastrectomy and quality of life. World J Surg. 1995;19(4):558-564. doi:10.1007/BF00294722

68. Hebbard PC, Macmillan A, Huntsman D, et al. Prophylactic total gastrectomy (PTG) for hereditary diffuse gastric cancer (HDGC): the Newfoundland experience with 23 patients. Ann Surg Oncol. 2009;16 (7):1890-1895. doi:10.1245/s10434-009-0471-z
69. Hallowell N, Lawton J, Badger S, et al. The psychosocial impact of undergoing Prophylactic Total Gastrectomy (PTG) to manage the risk of Hereditary Diffuse Gastric Cancer (HDGC). J Genet Couns. 2017;26(4):752-762. doi:10.1007/s10897-016-0045-8

70. Kaurah P, Fitzgerald R, Dwerryhouse S, Huntsman DG. Pregnancy after prophylactic total gastrectomy. Fam Cancer. 2010;9 (3):331-334. doi:10.1007/s10689-009-9316-y

71. Simpson JL. Preimplantation genetic diagnosis at 20 years. Prenat Diagn. 2010;30(7):682-695. doi:10.1002/pd.v30:7

\section{Publish your work in this journal}

Cancer Management and Research is an international, peer-reviewed open access journal focusing on cancer research and the optimal use of preventative and integrated treatment interventions to achieve improved outcomes, enhanced survival and quality of life for the cancer patient.
The manuscript management system is completely online and includes a very quick and fair peer-review system, which is all easy to use. Visit http://www.dovepress.com/testimonials.php to read real quotes from published authors. 